\title{
Numerical and Experimental Design Study of a Regenerative Pump
}

\author{
Francis J Quail $^{\mathrm{a}}$, Matthew Stickland ${ }^{\mathrm{a}}$ and Thomas Scanlon ${ }^{\mathrm{a}}$ \\ ${ }^{a}$ Department of Mechanical Engineering, University of Strathclyde, Glasgow, G11XJ, Scotland.
}

\begin{abstract}
This paper presents the use of a commercial CFD code to simulate the flow-field within the regenerative pump and compare the CFD results with new experimental data. Regenerative pumps are the subject of increased interest in industry as these pumps are low cost, low specific speed, compact and able to deliver high heads with stable performance characteristics. The complex flow-field within the regenerative pump represents a considerable challenge to detailed mathematical modelling. This paper also presents a novel rapid manufacturing process used to consider the effect of impeller geometry changes on the pump efficiency. Ten modified impeller blade profiles, relative to a standard radial configuration, were evaluated. The CFD performance results demonstrate reasonable agreement with the experimental tests. The CFD results also demonstrate that it is possible to represent the helical flow field for the pump which has only been witnessed in experimental flow visualisation until now. The ability to use CFD modelling in conjunction with rapid manufacturing techniques has meant that more complex impeller geometry configurations can now be assessed with better understanding of the flow-field and resulting efficiency.
\end{abstract}

Keywords: CFD, regenerative pump, helical flow, impeller PACS: 47.85.Kn

\section{INTRODUCTION}

Pumps are the single largest user of electricity in industry in the European Union, and of those pumps, centrifugal pumps represent some $73 \%$ of all pump types [1]. The regenerative pump like the centrifugal pump is a kinetic pump however the regenerative pump can in many applications offer a more efficient alternative [2]. There is limited published data and insufficient design guiding criteria to allow more intuitive industrial selection of this pump type, particularly to meet more stringent European pump selection criteria [3].The existing numerical models are limited in representing the complex flow-field within the pump and require significant experimental correction. Most of the theories presented, relied on assumptions not based on detailed measurements or precise CFD modelling. The previous published theories rely on experimental correction that take no spanwise account of flow (one dimensional). To date, the most fruitful research work has come from corresponding flow visualisation studies [4-7]. This paper considers a numerical and experimental analysis of a regenerative pump to simulate the flowfield and match pump performance. This paper also considers the effect of impeller blade geometry changes on the pump efficiency. This paper presents the use of a commercially available solver; FLUENT [8], in conjunction with new experimental testing to resolve the 
flowfield. The main characteristic of regenerative pumps is the ability to generate high discharge pressures at low flowrates, and ability to operate with very small NPSH, [2]. Although the pump has other advantages over centrifugal type pumps the main challenge is to understand and improve the hydraulic efficiency, typically $35-50 \%$. The highest ever reported efficiency for the regenerative pump of 50\% [9].

The ability to apply CFD to represent the 3D flow domain within the pump would represent a significant advance on current 1D mathematical models. Until this point the best interpretation of the flowfield came from flow visualisation work for regenerative pumps. This paper describes the use of new experimental data to compare, not correct, with CFD numerical results, and to consider if this can be done across a range of performance points. The paper also investigates how representative the CFD model is of previously published flow visulisation studies.

\section{NOMENCLATURE}

$\begin{array}{lll}A & \text { Cross sectional area } & \left(\mathrm{m}^{2}\right) \\ C F D & \text { Computational fluid dynamics } & \\ C O^{2} & \text { Carbon dioxide } & \\ D & \text { Impeller diameter } & (\mathrm{m}) \\ H P C & \text { High performance computer } & \\ P & \text { Power } & (\mathrm{kW}) \\ Q & \text { Volume flow rate } & \left(\mathrm{m}^{3} / \mathrm{s}\right) \\ R e \equiv \rho U A / \mu & \text { Reynolds number } & \\ \mathrm{U} & \text { Mean fluid velocity } & (\mathrm{m} / \mathrm{s}) \\ \mu & \text { Dynamic viscosity } & \left(\mathrm{N} \cdot \mathrm{S} / \mathrm{m}^{2}\right) \\ \rho & \text { Density } & \left(\mathrm{kg} / \mathrm{m}^{3}\right) \\ \mathrm{r} & \text { Impeller radius } & (\mathrm{m}) \\ \mathrm{H} & \text { Head } & (\mathrm{m}) \\ \mathrm{k} & \text { Turbulent kinetic energy } & \left(\mathrm{m}^{2} / \mathrm{s}^{2}\right) \\ \varepsilon & \text { Turbulent dissipation energy } & \left(\mathrm{m}^{2} / \mathrm{s}^{3}\right) \\ \mathrm{p} & \text { Pressure } & \left(\mathrm{kN} / \mathrm{m}^{2}\right) \\ \mathrm{N} & \text { Rotational speed } & \left(\mathrm{rev} / \mathrm{min}^{2}\right) \\ \mathrm{g} & \text { Gravitation acceleration } & \left(\mathrm{m} / \mathrm{sec}^{2}\right) \\ \mathrm{y}+ & \text { Boundary layer wall function } & \\ \eta & \text { Efficiency } & \\ \xi & \text { Experimental uncertainty } & \\ \omega & \text { Angular velocity } & (\mathrm{rad} / \mathrm{s}) \\ \varphi & \text { Flow Coefficient } & \\ \psi & \text { Head Coefficient } & \\ \mathrm{IP} & \text { Power Coefficient } & \\ \mathrm{T}_{\mathrm{i}} & \text { Turbulence Intensity Coefficient } & \end{array}$




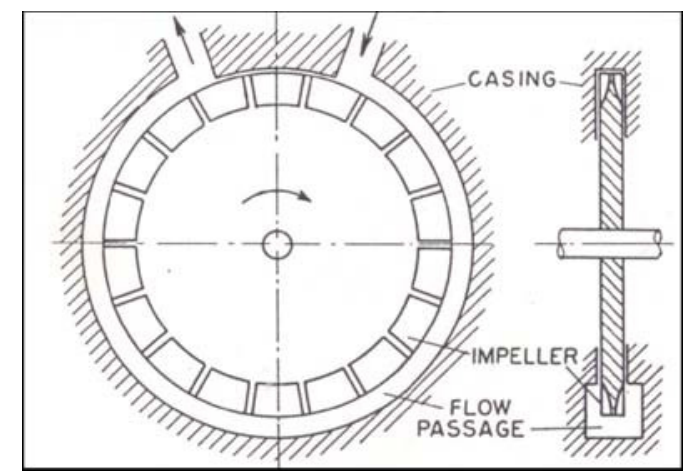

FIGURE 1. Regenerative Pump Schematic

\section{THE REGENERATIVE PUMP}

The pump uses an impeller with turbine-type blades mounted on the periphery, running in an annular channel surrounding the periphery of the impeller Fig. 1. In the standard design, the impeller has radial teeth machined into the impeller periphery and the fluid passes through an open annular channel and circulates repeatedly through the impeller vanes Fig. 2.

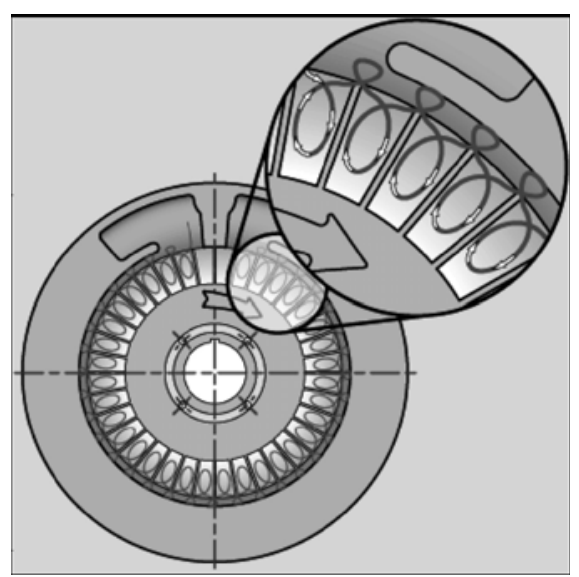

FIGURE 2. Regenerative Pump Helical Flowpath

This paper also considers the effect of impeller blade geometry changes to the pump efficiency from a standard radial blade configuration Fig. 3. The regenerative pump is sometimes also referred to as a peripheral pump, turbulence pump, friction pump, turbine pump, drag pump, side channel pump, traction pump or vortex pump.

The suction region is separated from the discharge region by a barrier on the casing known as a Stripper. The repeated fluid circulation during the flow process or 'multistaging' principally allows regenerative pumps to generate high heads at relatively low specific speeds. In spite of having operating characteristics that mimic a positive displacement pump, (power directly proportional to head, with maximum 
power required at shutoff, and a steep head-capacity curve), the regenerative pump is a kinetic pump. That is kinetic energy is imparted to the fluid by the series of impulses given to the fluid by the rotating impeller blades. At inlet the fluid splits to both sides of the impeller and continuously circulates between the blades and the channel.

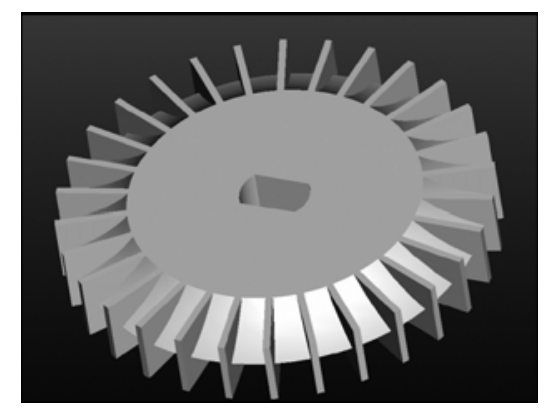

FIGURE 3. Regenerative Pump Impeller

When the circulation flow in the impeller and the peripheral flow in the channel unite the momentum exchange that takes places develops a helical or corkscrew fluid motion [10]. The regenerative pump will develop significantly higher heads than a centrifugal pump with comparable impeller size [2]. The objective of the numerical approach is to predict performance over a range of running conditions that can be validated by experimental testing. Furthermore a suitably validated CFD model provides the opportunity to demonstrate flow field representation without the significant expense of such experimental flow visualisation.

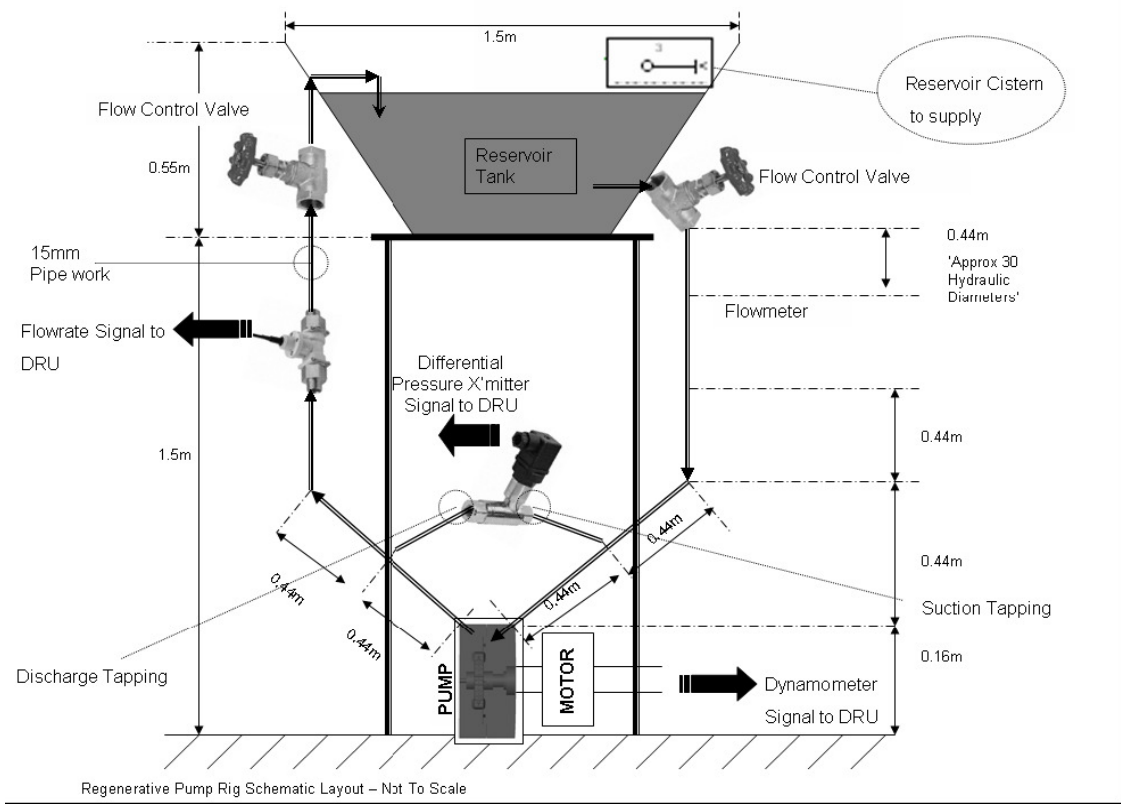

FIGURE 4. Regenerative Pump Rig Schematic 


\section{EXPERIMENTAL PROCEDURE}

The experimental rig, arrangement, Fig. 4, incorporates a reservoir tank which stores and ultimately receives the working fluid, in this case water. The fluid is drawn to the pump from the tank via a flow control valve.

The fluid flowrate is measured using a Hall Effect turbine flowmeter, $(0-30 \mathrm{l} / \mathrm{min})$, situated downstream of the flow control valve and upstream of the pump. The pump itself was driven by a $3 \mathrm{~kW}$ induction motor operating at a constant speed of $3000 \mathrm{rpm}$. The motor housing is coupled to a dynamometer containing a load cell to measure strain and hence indicate input torque to be used in the pump efficiency calculations, Fig. 5.

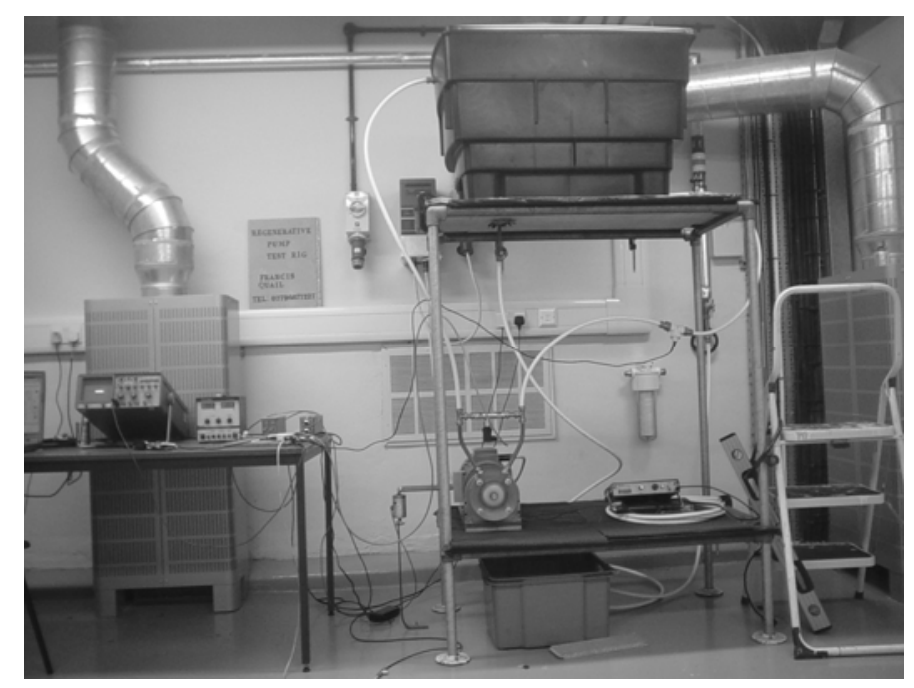

FIGURE 5. Experimental Test Arrangement

The loadcell (using a Wheatstone bridge arrangement) strain measurement has been calibrated against force and is converted to a reaction torque $(0-20 \mathrm{Nm})$. The pump differential pressure was measured using a high performance millivolt output pressure transducer $(0-5$ Bar). The fluid flowrate is adjusted via a flow control valve metering the flow to allow a range of measurements to be taken to develop a running characteristic. This enables a range of flows and the corresponding pump inlet / outlet pressures and input torque values to be measured. The test impeller had 30 blades of width $12 \mathrm{~mm}$ and diameter $74.5 \mathrm{~mm}$. The pump is of double suction shape designed with alignment of the blades to balance axial thrust Fig. $(1,3)$. In this design the impeller has radial teeth or vanes machined into each side at its periphery. The measurements are collected using a data acquisition unit and pump characteristic flow, head, power and efficiency coefficients can be calculated as expressed in conventional dimensionless terms Eq. (1-5). 


$$
\begin{aligned}
& \psi=\frac{g H}{\omega^{2} D^{2}} \\
& \varphi=\frac{Q}{\omega D^{3}} \\
& I P=\frac{P}{\rho \omega^{3} D^{5}} \\
& \eta=\frac{Q \Delta p}{P} \\
& T_{i}=\frac{k}{\omega^{2} r^{2}}
\end{aligned}
$$

The dimensionless plots are used to illustrate the regenerative pump is a hydrodynamic unit obeying the same similitude laws as centrifugal and axial pumps, turbines and compressors.

To estimate the overall experimental uncertainty the root of the sum of the squares is used $\xi_{R}$, where $\mathrm{R}$ is the dependent variable of interest, $\mathrm{i}$ is the index representing the measured variable and $\delta_{i}$, the sensitive coefficient of $\mathrm{R}$ with respect to $\mathrm{X}_{\mathrm{i}}$ Eq. $(6,7)$ [11].

$$
\begin{gathered}
\xi_{R}= \pm \sqrt{\sum\left(\delta_{i} \xi_{x i}\right)^{2}} \\
\delta_{i}=\frac{\delta R}{\delta X_{i}} \\
\xi_{\eta}= \pm \rho g\left[\left(\xi_{Q} \frac{H}{P}\right)^{2}+\left(\xi_{H} \frac{Q}{P}\right)^{2}+\left(\xi_{P} \frac{Q H}{P^{2}}\right)^{2}\right]^{\frac{1}{2}}
\end{gathered}
$$

For a typical case of the regenerative pump, a 5\% error was determined for the flowrate, a $0.6 \%$ error for the head and $4.3 \%$ error in the power calculation. Applying Eq. 8 , this equates to a pump efficiency error of $6.6 \%$. The random scatter was evaluated from repeatability tests and sensitivity analyses. The systematic inaccuracy due to aggregate systematic errors in transducers and changes in performance due to build-to-build differences are difficult to evaluate [12]. To achieve this it is essential that the data acquisition system incorporates procedures which evaluate the quality of the data as it is acquired. This allowed comparison of the actual data with expected, and when necessary analysis of the raw measurements to verify accuracy.

In the rig arrangement, Fig. $(4,5)$, measures where taken to minimise effects which could reduce the inlet pressure to the pump. Selection of optimal inlet line length, and 
bore were considered as well as pump elevation and upstream discontinuities that affect inlet pressure. Regenerative pumps, typically, require lower net positive suction heads than other kinetic pumps, e.g. centrifugal pumps [2].

\section{MANUFACTURE}

Until now regenerative pump impellers have retained a fairly basic geometric configuration with simple radial vanes machined into the impeller, [13]. The purpose of this paper was to find a rapid prototyping technique that was robust enough to produce, for the first time, more complex regenerative pump impellers for conducting experimental tests in conjunction with CFD numerical analysis [14]. Four methods of rapid manufacturing where considered for impeller production. Ultimately a combination of Fused deposition modelling (FDM) Fig.6, followed by Room temperature vulcanisation (RTV) Fig. 7 was used that produced impellers robust enough to permit testing, Fig 8.

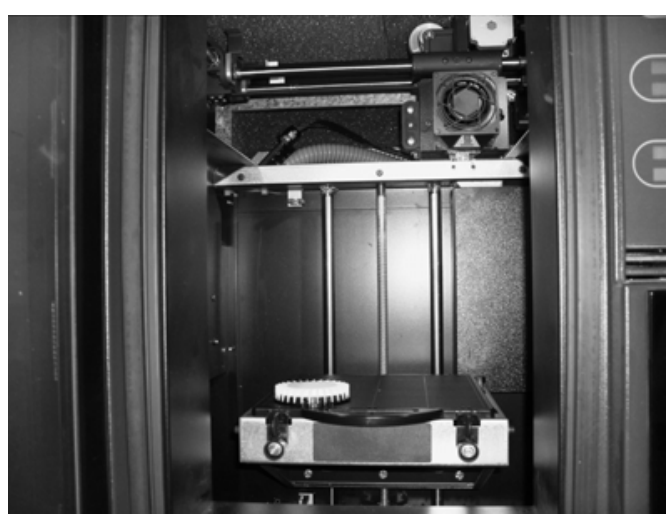

FIGURE 6. Fused deposition modeling regenerative impeller

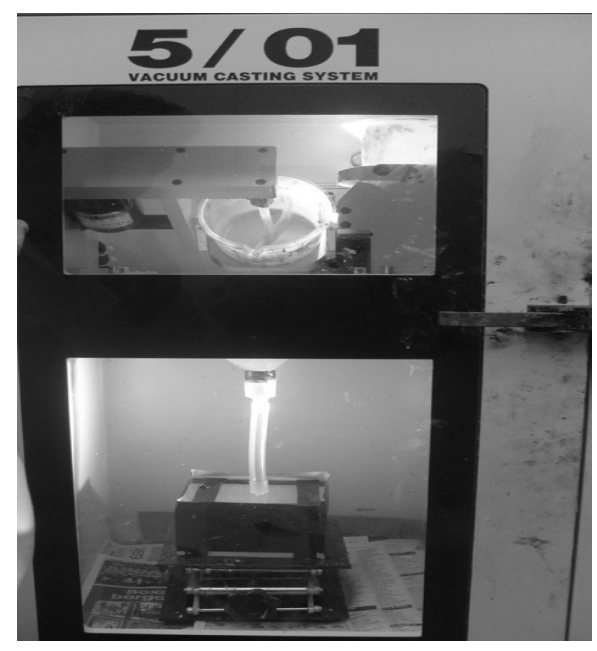

FIGURE 7. Room temperature vulcanisation regenerative impeller 


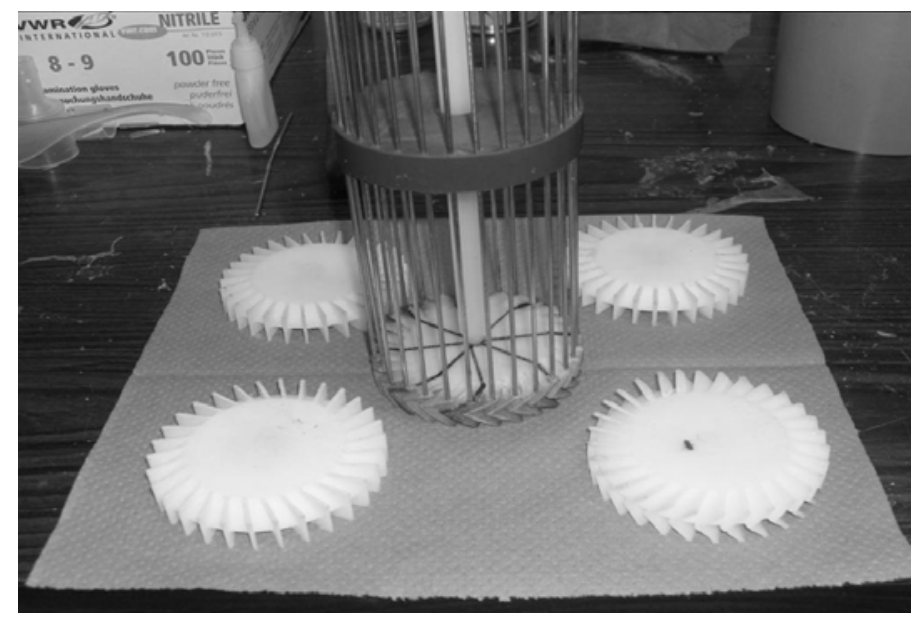

FIGURE 8. Rapid prototyping regenerative impellers

\section{CFD MODELLING}

Fluent Best Practices for Rotating Machinery, [15], recommends that for complex turbomachinery geometry, a non-conformal hybrid hexahedral / tetrahedral mesh is appropriate where the rotation of the rotor is treated as a steady-state in a multiple reference frame model (MRF). In the case of the regenerative pump separate meshes were generated for the rotating impeller Fig.9. and the stationary casing Fig.10. The pump flow was then solved in local rotating reference frames where fluxes are locally transformed from one frame to another at the pump zone interfaces.

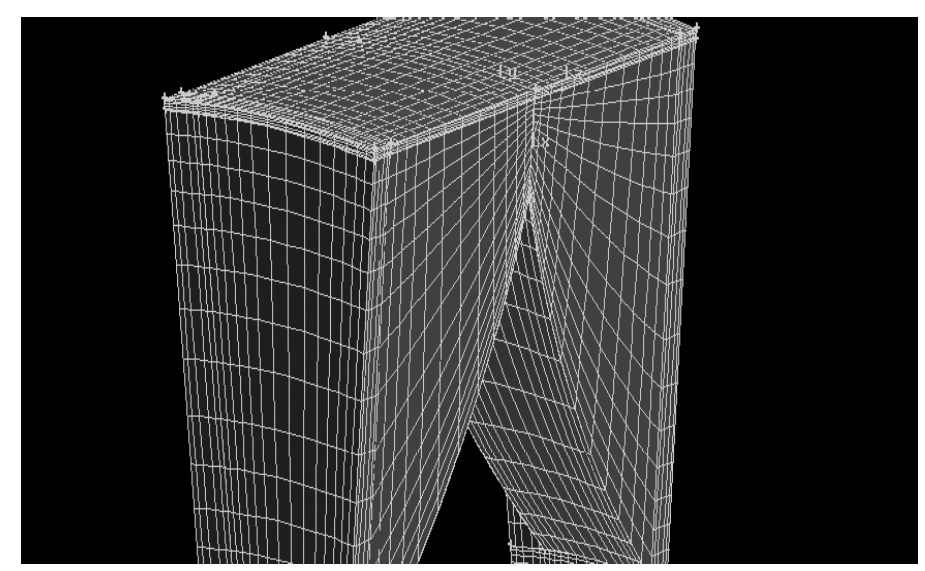

FIGURE 9. Impeller Fluid Region Hex Mesh 


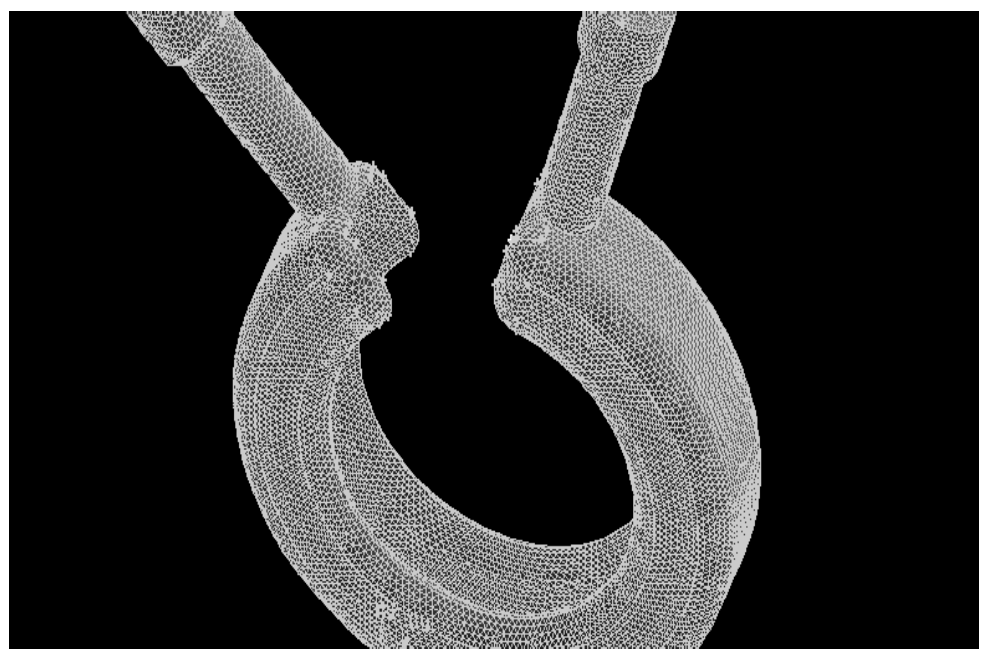

FIGURE 10. Casing Fluid Region Tet Mesh

For the regenerative pump application a pressure-based solver was chosen as the current analysis only considers incompressible flow. The velocity formulation selected was to use Absolute Velocity Formulation (AVF) as the fluid inflow comes from a stationary domain. In this case absolute total pressure was measured during the regenerative pump testing. The MRF model is appropriate for incompressible flows as the flowfield responds instantly to changes in rotor position. A different approach would be required, to consider compressibility of the fluid e.g. in regenerative blowers, [16,17], but for the current analysis where the fluid is treated as incompressible then use of MRF at multiple fixed rotor positions is a suitable and a recommended approach, [18-20].

For modeling turbulence, realizable $\mathrm{k}-\varepsilon$ was chosen, [20,21], for the regenerative pump as it is suitable for complex shear flows involving rapid strain, swirl, vortices and locally transitional flows (boundary layer separation and vortex shedding). Unlike many pump cases the clearances are very small between the impeller and the casing in the regenerative pump stripper region. In considering the above there is a balance to achieve good convergence, satisfying the performance matching and in modeling turbulence the mesh should be made either coarse or fine enough to prevent the walladjacent cells from being placed in the buffer layer $\left(\mathrm{y}^{+}=5-30\right)$. Using excessive stretching in the direction normal to the wall was avoided. It is important to have at least a few cells inside the boundary layer and for the pump this was kept to a minimum of 5 cells. For the wall functions, each wall-adjacent cell's centroid should be located within the log-law layer, $30<\mathrm{y}+<300$. A $y+$ value close to the lower bound $(\mathrm{y}+\sim 30)$ was sought. When using adaption this can result in large cell size changes which was to be avoided. In Fluent application briefs [18-20] MRF simulations made use of tetrahedral and hybrid meshes of between 1 million cells to 2.4 million cells.

It is essential to minimize cell skewness and aspect ratio. Skewness was kept below 0.9 and aspect ratios of greater than 5:1 are not recommended in FLUENT turbomachinery applications, [15]. Initially the model was a complete tetrahedral mesh 
(impeller and casing) which resulted in a 753,000 cell model; however the impeller was decomposed to prevent numerical error (false diffusion) across the flowfield and for greater post-processing control (impeller surfaces plots). The grids were adapted until there was only small differences in $(<1 \%$ change) parameters. Four adapted grid sizes where assessed, 400,000; 800,000; 1.6 million, 1.9 million and 2.4 million cells. Grid independence was established at around 1.9 million cells. The results where comparable in accuracy with those published by FLUENT [18-20]. There was no significant change in the solution at around 1.9million cells, and as grid independence is of importance, quality of the mesh (particularly in the buffer region) and performance results are also important.

Most of the published data until now suffers from two fundamental problems which limit their use as a design tool. The first is a reliance on empirically derived loss factors which are not directly related to design parameters and the second defect is that they are an essentially one dimensional tool and take no account of spanwise variation, [23].

\section{RESULTS}

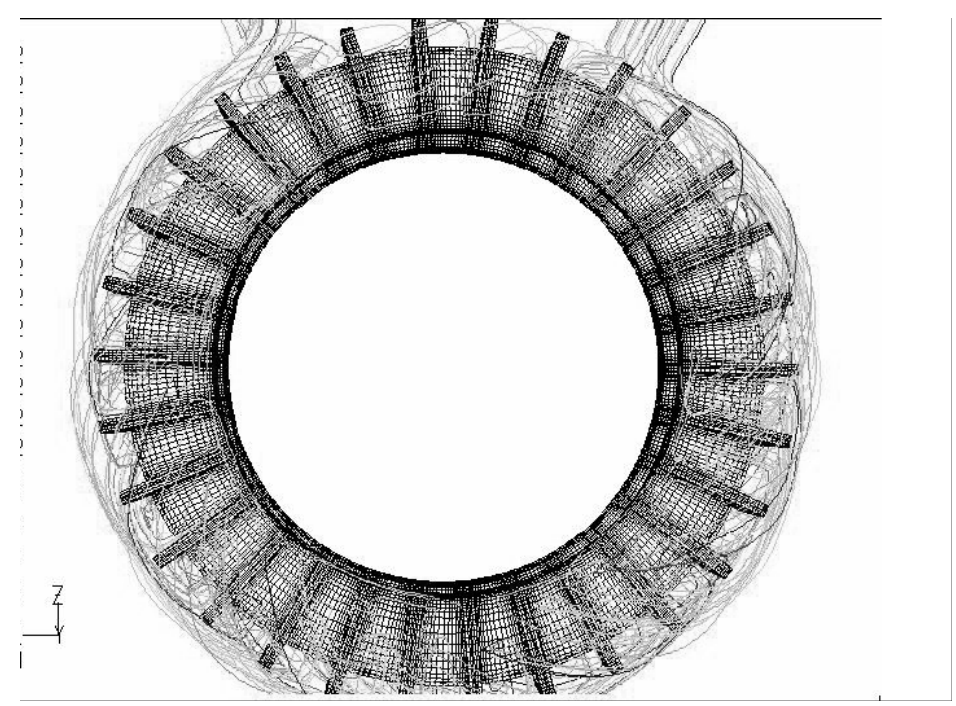

FIGURE 11. Regenerative Pump Helical Pathlines Plot

Considering the numerical modelling approach, the ability to successfully capture the flow field in a manner that has not been achieved since the flow visulisation experiments is indicated in Fig. 11 and Fig. 12. The pathlines plot indicates the helical or corkscrew motion that occurs within the pump. The helicity can be displayed in an iso-surface section through the impeller and channel fluid region to depict the strong helicity gradient at the interface region between the impeller and channel region. 


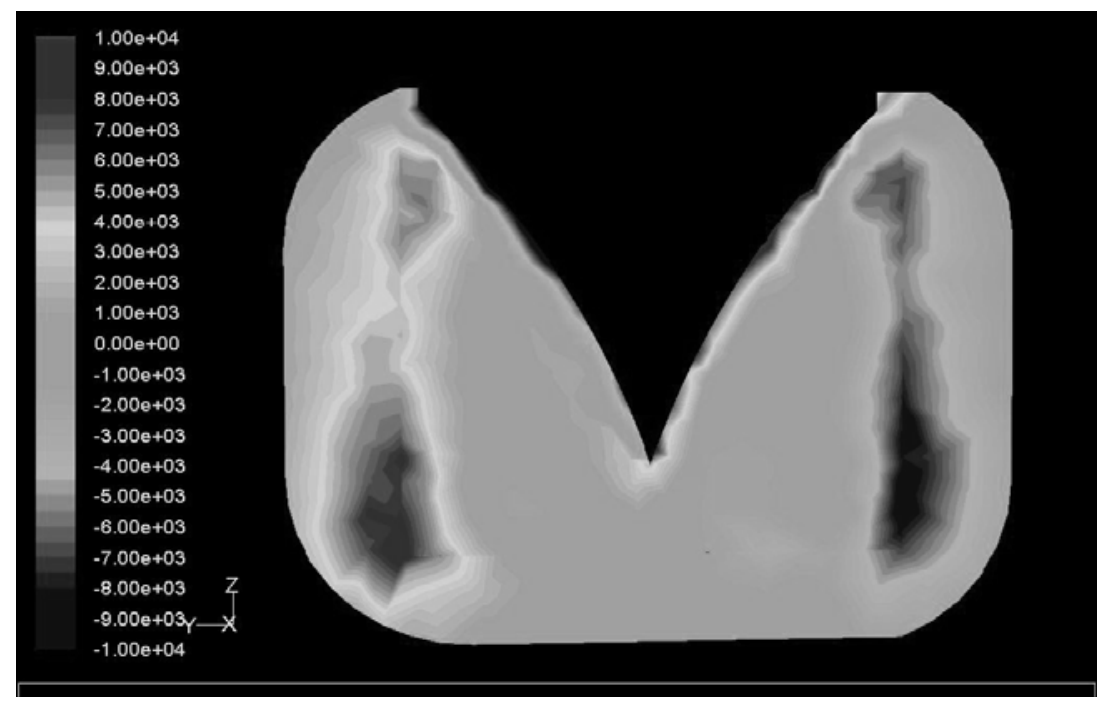

FIGURE 12. Regenerative Pump Helicity Contours

When the flow in the side channel unites with the circumferential flow in the impeller the momentum exchange that occurs, [24] is the mechanism which initiates and sustains the helical fluid flow, [10]. Experiments conducted, [4-7], used small thread probes at different points in the annular flow passage of the pump to determine the direction of the flow velocity. They were able to corroborate the helical streamlines when plotting the results. With decreasing flowrate, pump circulation is considerably increased reaching a maximum as the flow from the pump is reduced [24]. Previous work, [25] that does not describe the helical flow nature instead conclude a constant circulation rate with reducing the flowrate. These theories conclude that the circulation is only dependant on the resistance of the flow in the side channel and the impeller and is independent of the pressure in the working channel.

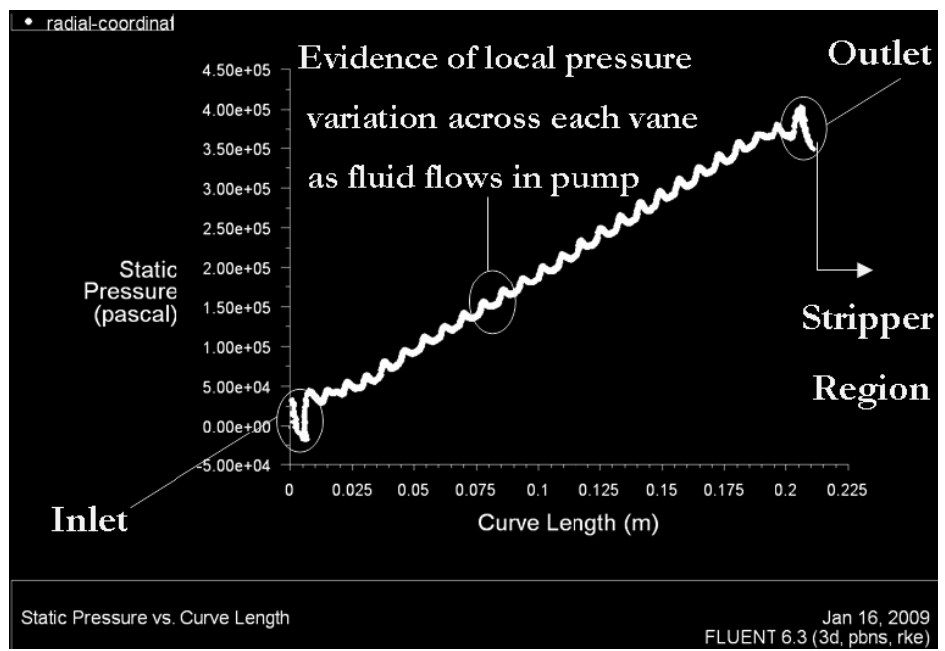

FIGURE 13. Local Pressure Variation Through Pump Working Section 
The current study indicates that in fact, as demonstrated in Fig.13, local pressure variations occur across each stage rise of the pump. The static pressure varies both in the channel and the impeller as it decelerates and accelerates in the pump as it makes a helical flow path through the pump. This understanding is limited in the typical straight linear representation of pressure distribution presented, [26], Fig. 14. Where Curve length $=$ Circumferential position around pump.

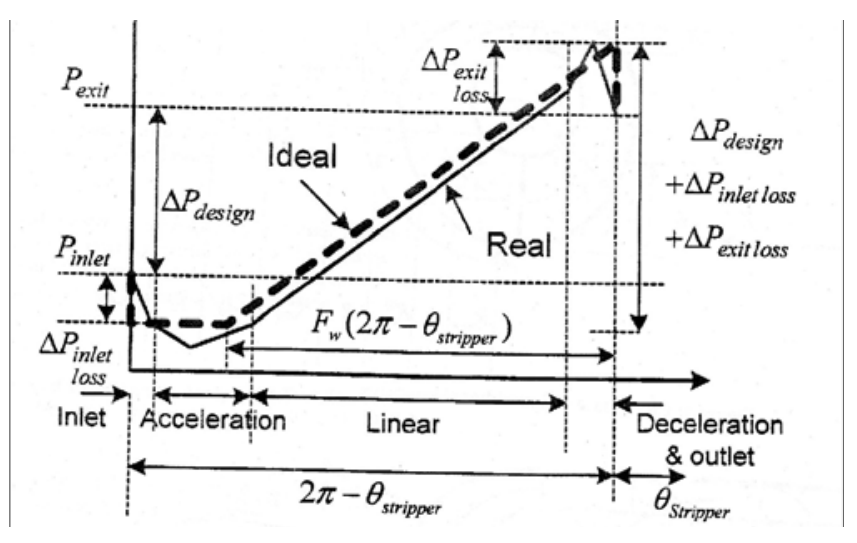

FIGURE 14. Local Pressure Variation through Pump Working Section [26]

It is not only in the flow visulisation that the CFD approach is beneficial in extending the knowledge of the flow physics, the ability to predict the performance of the pump in the model without the need for experimental correction factors being applied is clear in Fig.15. The reasonable concurrence between the experimental results and the CFD predictions is indicated in Fig 16.

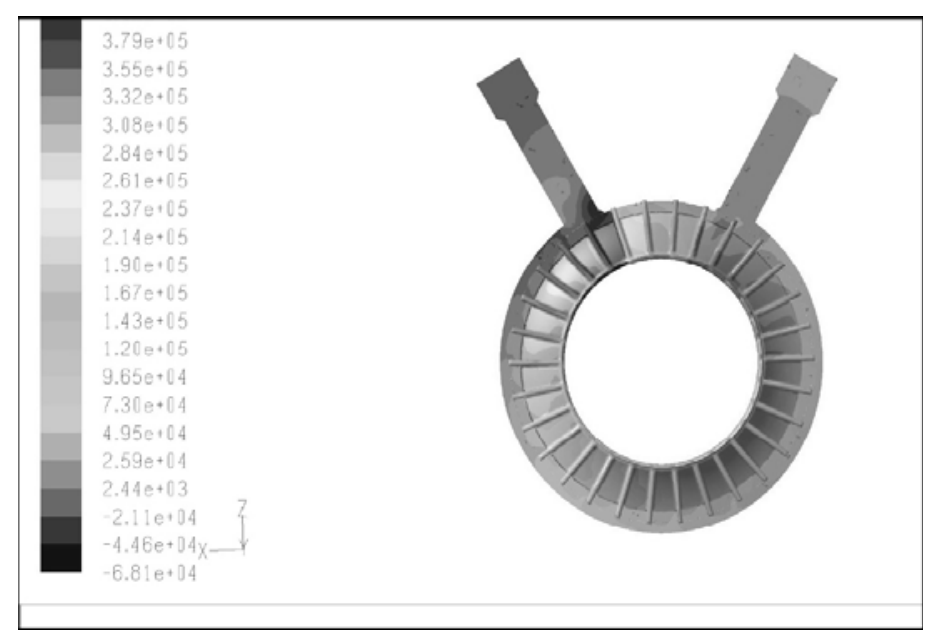

FIGURE 15. Regenerative Pump Pressure Contours 


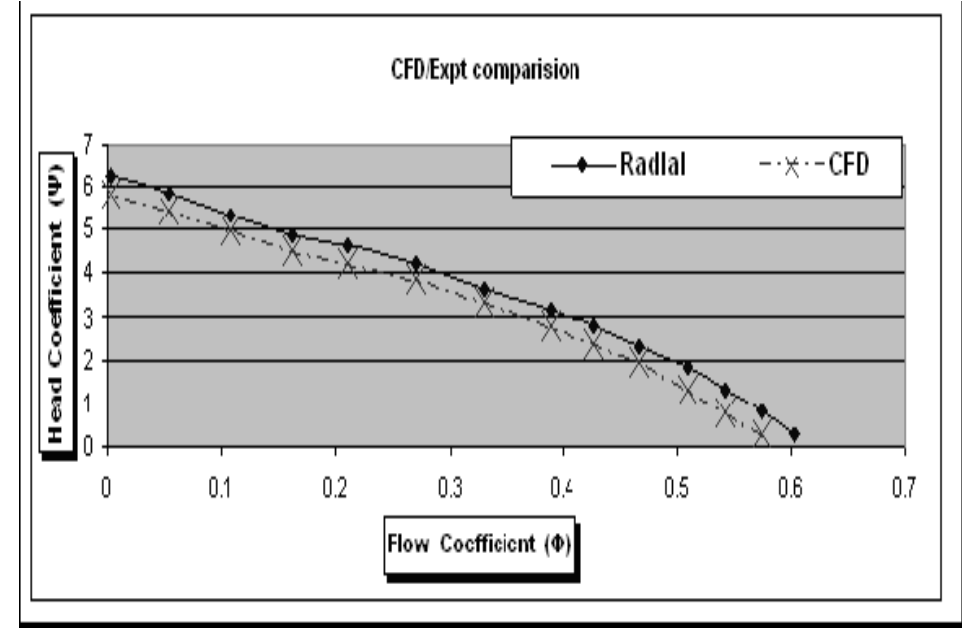

FIGURE 16. Head Coefficient vs. Flow Coefficient

The 'multistaging' effect that allows regenerative pumps to generate high heads at relatively low speeds is not only captured but the efficiency challenge for the pump can be seen where the measured efficiency, Fig 16, has matched the highest ever reported efficiency for this pump type, [9].

When considered against centrifugal devices of similar specific speed the efficiency of the regenerative pump can in many applications be higher, [2]. The benefit of the regenerative pump in the ability to operate at low NPSH is indicated in, Fig 17.

An iso-plot of typical pressure contours shows the rapid rise in pressure gradient, Fig 17, within pumps of this type.

The rise follows the established characteristic of a regenerative pump. In Fluent application briefs [18-20] water pump MRF simulations made use of tetrahedral and hybrid meshes of similar scale. In the current study the experimental results and the CFD predictions are within 3\%, indicating that the meshing strategy was reasonable, [12]. The examples referenced above $[18,20]$ at best achieved a $7 \%$ matching. Typical experimental spread even in calibrated data was found to be around $6 \%$ indicating a reasonable matching procedure presented in the current paper.

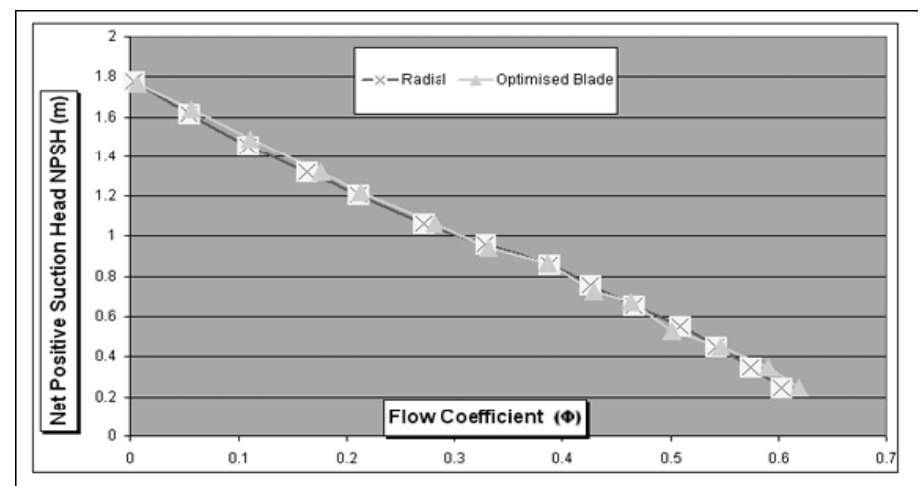

FIGURE 17. NPSH vs. Flow Coefficient 
Most authors have concluded that substantial efficiency and performance improvement would be attained with better understanding of the flowfield in the regenerative pump [27-29]. Whilst the current work indicates a reasonable concurrence with experimental data Fig. 16 it is important to comment on the possible sources of error.

In matching there is often some simplification of geometry, or the mesh may be left relatively coarse in the tip region, and other smaller features such as fillets may not be fully represented. The simplification of the true geometry, due to difficulties in obtaining grids, or restrictions on the numbers of nodes which may be used due to the limitations in processing power, leads to unquantifiable errors. These errors could become significant relative to the performance increments now being sought. There is a trade off to ensure mesh quality, near wall modelling, and the computational cost of the mesh. MRF may be difficult to solve because of large flow gradients resulting from the rotation of the fluid domain. MRF grid interfaces introduce some error due to the nature of the MRF approximation (i.e. local transfer of flow properties across the interface with no account for grid motion). Steady-state simulation changes in relative position between stationary and rotating meshes (e.g., interaction and interference) are not accounted for in the MRF model. It is not accurate if recirculation exists at the interfaces. This is known to under-predict the flow rate (1-3\%) due to losses, [8].

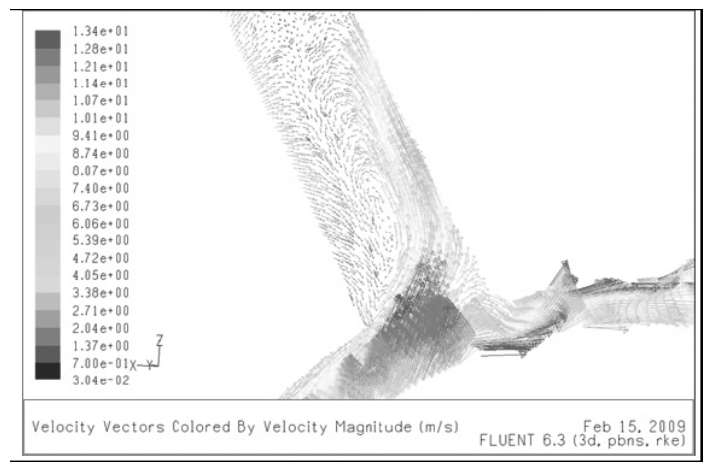

FIGURE 18. Velocity vectors regenerative pump

Accuracy and repeatability are major and inescapable issues in testing and have been considered in the experimental section of this paper. Pump efficiency error for the indicated case can be of the order of $6.6 \%$. Whilst accuracy is an issue in CFD repeatability should not be, given the same solution starting conditions. The mesh definition and quality (clustering, orthogonallity, cell aspect ratio, etc.) have a considerable influence on accuracy; with highly skewed cells in particular have a large impact [30]. Geometric features of the impeller blade were modified after an analysis of flow alignment carried out by the author [14] Fig's. 18, 19.

The ability of CFD to be used to assess performance and for example losses within the flowfield is assisting the design optimisation process for this pump type [20]. 


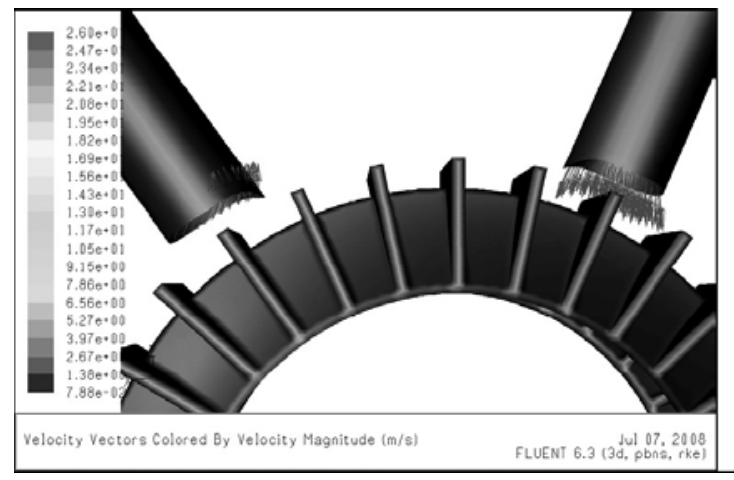

FIGURE 19. Velocity vectors regenerative pump

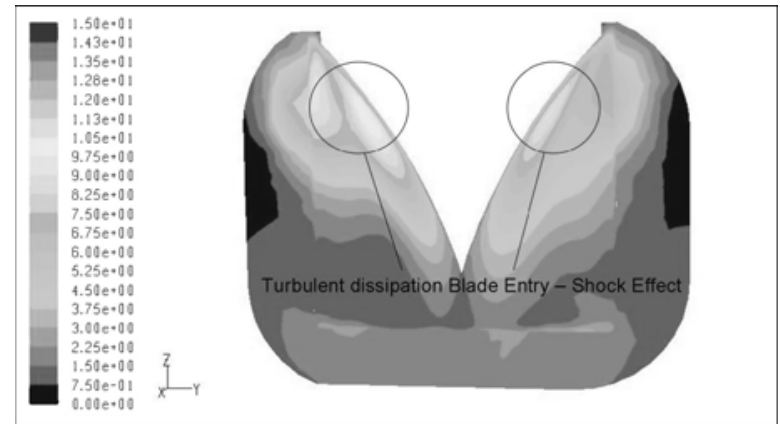

FIGURE 20. Turbulent Kinetic Energy Distribution

\section{CONCLUSIONS}

There are a number of conclusions which may be drawn with regard to effectively matching the regenerative pump CFD model with the experimental data. CFD results obtained represent a reasonable match both in performance and flow visulisation experiments and are being utilised to focus investigation for unit performance improvement. As the capabilities of CFD continue to develop, it is to be expected that the uncertainties associated with CFD prediction should also reduce. There is a need for significant developments in instrumentation technology and manufacturing approaches which enable detailed data to be acquired over large regions at higher accuracy, and strength at a reasonable cost.

This work has been useful to not only benchmark current regenerative pump design, but gives confidence in the ability of CFD optimisation for the design to increase the performance of the pump in the future. The ability of the CFD to establish a reasonably good representation of the pump under steady state incompressible conditions is the starting point to consider more complex arrangements under a more varied performance environment. Advances in both Rapid Manufacturing and Computational Fluid Mechanics will assist such optimisation in the future. 


\section{REFERENCES}

1. IMechE, Energy Saving in pumps and Pumping, Fluid Machinery Group Symposium (2007)

2. S, Mueller, Consider regenerative pumps for low flow/low NPSH applications. Hydrocarbon processing pg 55-57 (2004)

3. European Parliament, Establishing a framework for the setting of ecodesign requirements for energy using products, Directive 2005/32/EC (2005)

4. H. Engels, Investigations of Ring pumps, Tech Hoch Hannover. (1940)

5. J. Bartels, Performance of a peripheral pump -Associate Professor, Polytechnic Institute of Brooklyn (1947)

6. L. Lazo, T. Hopkins, Theoretical and experimental analysis of a regenerative turbine pump, Massachusetts Institute of Technology (1953).

7. G.F. Lutz, Experimental Investigation of the pressure distribution in a regenerative turbine pump, Massachusetts Institute of Technology (1953).

8. FLUENT version 6.3.26.: (C ANSYS Inc. All Rights Reserved (2006)

9. E. Crewdson, Water-ring self-priming pumps. -Proceedings of the Institution of Mechanical Engineers Vol. 170 No. 13, pp. 407-415. (1956).

10. F.J Quail. Design optimisation of a regenerative pump using numerical and experimental techniques" - University Of Strathclyde - (2009) PhD Thesis

11. L Kirkup, An introduction to the analysis of presentation of data, Experimental Methods - John Wiley and sons (1994).

12. G. Woollatt, D. Lippett, P.C. Ivey, P. Timmis, B.A.Charnley, The Design, Development and Evaluation of 3d Aerofoils for High Speed Axial Compressors, Part 2: Simulation and Comparison with Experiment", ASME TURBOEXPO, Paper GT-2005-Nevada. (2005)

13. A. Engeda, Flow analysis and design suggestions for regenerative flow pumps, ASME FEDSM2003-45681 (2003)

14. F.J.Quail, T.J.Scanlon, M. Stickland, Rapid Manufacturing Technique used in the Development of a Regenerative Pump Impeller, Proceedings of World Congress On Engineering - London (2009)

15. FLUENT - Best Practices For Rotating Machinery (2006).: ( $)$ ANSYS Inc. All Rights Reserved

16. H. Sixsmith, H. Altmann, A Regenerative Compressor, Trans ASME Vol 99pp 637-647 (1977)

17. W, Hollenberg, J.H. Potter, An Investigation of Regenerative Blowers and Pumps- Trans ASME Vol $101 \mathrm{pp}$ 147-152 (1979)

18. FLUENT. Backward inclined Centrifugal Fan- Ex143 Application briefs from FLUENT - (2001)

19. FLUENT. Automotive Water Pump- Ex164 Application briefs from FLUENT - Courtesy of TESMA Engine Technologies (2001)

20. FLUENT. Mixed Flow Pump- Ex232 Application briefs from FLUENT - (2005)

21. P. Spalart, Trends in turbulence treatments, .AIAA paper (AIAA 2000-2306) (2000)

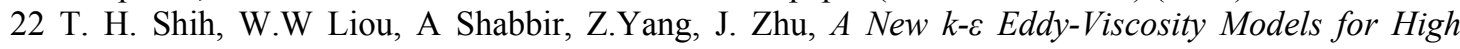
Reynolds Number Turbulent Flows-Model Development and Validation, Computers Fluids, 24 (3), pp. 227-238. (1995)

23. H.W. Iverson, Performance of the Periphery Pump, Trans ASME Vol 77 pp 19-28 (1955)

24. W.A.Wilson, M.A. Santalo, J.A. Oelrich, $A$ Theory of the fluid dynamic mechanism of regenerative pumps, Trans. ASME Vol 77 PP1303-1316 (1955)

25. Y. Senoo, Theoretical research on Friction Pump, Institute of Fluid Engineering Vol 5 No1 pp 2348 (1948)

26. M. Raheel, A. Engeda Systematic design approach for radial blade regenerative turbomachines Journal for Propulsion and Power Vol. 21 (2005)

27. G. Pfleiderer, Pumps for liquids and gases, Springer - Verlag, 5th edition (1961)

28. M. Badami, Theoretical and experimental analysis of traditional and new peripheral pumps" SAE Technical Papers Series, No 971074 (1997)

29. J.W. Song, A. Engeda, M.K. Chung, Modified theory for the flow mechanism in a regenerative flow pump, Proceedings IMECHE, Vol 217 (2003) Power and Energy

30. C.H. Hirsch, CFD Methodology and Validation for Turbomachinery Flows, Paper 4 in: AGARDLS-195, Turbomachinery Design Using CFD. (1994) 